\title{
Evolutionary models of zero metallicity stars
}

\author{
Paola Marigo, Cesare Chiosi, Léo Girardi, and Tiziana Sarrubbi \\ Department of Astronomy, University of Padova, Vicolo dell'Osservatorio 5, 35122 \\ Padova, Italy
}

\begin{abstract}
We present new evolutionary models for zero-metallicity stars, covering a large range of initial masses (from 0.8 to $100 M_{\odot}$ ). Models are computed with overshooting from stellar cores and convective envelopes, and assuming mass-loss from the most massive stars. We discuss the main evolutionary features of these stars, and provide estimates of the amount of newly-synthesized elements dredged-up to the stellar surface, and possibly lost by stellar winds from the most massive stars. Full details of these models will be given in Marigo et al. (2000, in preparation).
\end{abstract}

\section{Model prescriptions}

In our computations we adopt an initial helium content $Y=0.23$. We consider the complete sets of reactions for the pp-chain and $\mathrm{CNO}$ tri-cycle, and the most important alpha-capture reactions for heavy elements up to $\mathrm{Mg}$. Nuclear rates are taken from Caughlan \& Fowler (1988). The nuclear network is implicitly solved for all the considered $\mathrm{H}$ - and He-burning reactions, and without any additional assumption for nuclear equilibria. Overshooting from stellar cores is applied according to the Bressan et al. (1981) formalism. Massloss from massive stars $\left(M \geq 10 M_{\odot}\right)$ is described according to Chiosi (1981). Stellar evolution is calculated throughout the nuclear H-burning phase up to the He-flash at the RGB tip for low-mass models, and throughout nuclear $\mathrm{H}$ - and He-burning phases up to the beginning of the TP-AGB phase and carbon ignition for intermediate- and high-mass models, respectively.

\section{Evolutionary features}

Figure 1 presents the evolutionary tracks in the H-R diagram. The appearance of short-lived loops is caused by the ignition of the 3- $\alpha$ reaction during core and/or shell H-burning, leading to the first activation of the CNO-cycle. In the case of low-mass stars $\left(0.9 M_{\odot} \lesssim M \lesssim 1.2 M_{\odot}\right)$ a loop develops near the end of central H-burning $\left(X_{\mathrm{c}} \sim 0.01\right)$, whereas for more massive stars $\left(2.5 M_{\odot} \lesssim M \lesssim 6 M_{\odot}\right)$ a similar feature also occurs at the formation of the He-shell.

As far as the critical stellar masses for non-degenerate nuclear ignition are concerned, we find that $M_{\mathrm{Hef}} \sim 1.1 M_{\odot}$ corresponds to the minimum initial mass for a star to avoid the He-flash, and $M_{\mathrm{up}} \sim 6.0-7.0 M_{\odot}$ the minimum initial mass for a star to avoid carbon deflagration. 


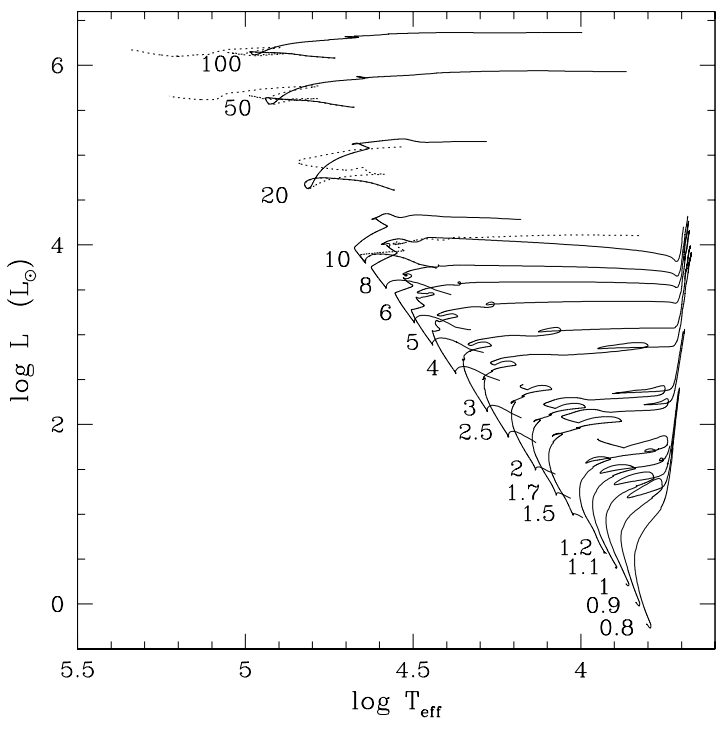

Fig. 1. HR diagram for the complete set of $Z=0$ evolutionary tracks, from 0.8 to 100 $M_{\odot}$. Solid line: evolution at constant mass; dotted line: evolution with mass-loss

\section{Surface chemical changes}

The first dredge-up is practically absent in all models. Only for stars with $M<1.0 M_{\odot}$ a very small amount (less than $0.003 M_{\odot}$ ) of He is dredged-up to the surface. The second dredge-up is experienced by stars with $1.5 M_{\odot}<$ $M<8 M_{\odot}$, being quite efficient in models with $M \geq 2.5 M_{\odot}$. It turns out that the surface composition is enriched almost only in He (reaching $Y=0.25$ -0.37 at increasing stellar mass) and negligibly in CNO elements $\left(10^{-16}\right.$ $10^{-8}$ in mass fraction).

Massive stars, with $10 M_{\odot}<M<100 M_{\odot}$, do not show any trace of surface chemical pollution due to dredge-up episodes. Only models calculated with mass-loss may expose nuclearly processed material to the surface. With the adopted prescription for mass-loss, stellar winds are never able to strip off H-exhausted layers in models with $M \leq 20 M_{\odot}$. In these cases, at most, the H-burning shell is eaten up, with consequent surface enrichment in $\mathrm{He}$ and small amounts of CNO. For more massive models with $M \geq 50 M_{\odot}$, wind stripping is able to reach the CO-enriched region left by convective Heburning. In these cases, the corresponding yields of $\mathrm{C}$ and $\mathrm{O}$ are considerable $\left(\sim 0.1-1 M_{\odot}\right)$.

\section{References}

1. Bressan A., Bertelli G., Chiosi C., (1981), A\&A 102, 25-30

2. Chiosi C., (1981), A\&A 93, 163-170

3. Caughlan G.R., Fowler W.A., (1988), Atomic Data Nucl. Data Tables 40, 283 